\title{
Analysis of strawberry ripening by dynamic speckle measurements
}

\author{
C. Mulone ${ }^{a, *}$, N. Budini ${ }^{a, b}$, F. M. Vincitorio ${ }^{a}$, C. Freyre ${ }^{a}$, A. J. López Díaz ${ }^{c}$, A. Ramil Rego ${ }^{c}$ \\ ${ }^{a}$ Universidad Tecnológica Nacional, Facultad Regional Paraná. Avda Almafuerte 1033, \\ E3100XAI, Paraná, Argentina; \\ ${ }^{b}$ Instituto de Física del Litoral (CONICET-UNL), Güemes 3450, S3000GLN, Santa Fe, \\ Argentina; \\ ${ }^{c}$ Universidade Da Coruña, Escuela Politécnica Superior, Av. Esteiro S/N, Ferrol, Spain.
}

\begin{abstract}
This work seeks to determine the age of a fruit from observation of its dynamic speckle pattern. A mobile speckle pattern originates on the fruit's surface due to the interference of the wavefronts reflected from moving scatterers. For this work we analyzed two series of photographs of a strawberry speckle pattern, at different stages of ripening, acquired with a CMOS camera. The first day, we took ten photographs at an interval of one second. The same procedure was repeated the next day. From each series of images we extracted several statistical descriptors of pixel-to-pixel gray level variation during the observation time. By comparing these values from the first to the second day we noticed a diminution of the speckle activity. This decay demonstrated that after only one day the ripening process of the strawberry can be detected by dynamic speckle pattern analysis. For this study we employed a simple new algorithm to process the data obtained from the photographs. This algorithm allows defining a global mobility index that indicates the evolution of the fruit's ripening.
\end{abstract}

Keywords: Dynamic speckle, biospeckle, mobility index, fruit maturation

\section{INTRODUCTION}

When an optically rough surface is illuminated with light having a high degree of coherence, such as one coming from a laser, the scattered light presents a particular intensity distribution making the surface appear to be covered with a fine granular structure. This structure, which consists of alternately dark and bright spots of variable shapes distributed in a random way, has no obvious relation to the macroscopic properties of the surface. ${ }^{1}$ The observation of dynamic speckle patterns variations on living cells, such as fruit cells, is a particular case of study known as biospeckle.

Speckle patterns serve to study some characteristics of diverse materials (organic and inorganic), which is generally carried out by two different methods: dynamic speckle analysis (or bispeckle) and digital speckle interferometry. In the latter method, two different states of a sample are compared having introduced an external perturbation between both states. On the contrary, the former method studies the evolution of a particular aspect of the sample under study. When it comes to biological samples, cell structures present changes on their size (or in their refraction indexes) that become evident due to an important temporal variation on the speckle pattern. ${ }^{1}$

When a laser with a wavelength above $600 \mathrm{~nm}$ is used, the speckle pattern does not only depend on the surface, but also on the inner tissues. This is due to the penetration depth of light near the bottom edge of the infrared in biological tissue. As an example, in the case of apple peel the penetration depth of light with a wavelenght above $600 \mathrm{~nm}$ is higher than $2 \mathrm{~mm} .{ }^{2}$ Pattern analysis must be done through a digital processing of the images taken with a CMOS camera. There are several methods to analyze temporal evolution of dynamic speckle patterns. ${ }^{3-6}$ In this work we present results obtained from the analysis of dynamic speckle patterns of strawberries through the application of a simple new algorithm based on the statistical parameters extracted from the acquired sequence of images.

\footnotetext{
*Corresponding author e-mail: ceciliamulone@gmail.com
} 


\section{METHODOLOGY}

\subsection{Optical methodology}

For illumination, a $50 \mathrm{~mW}$ HeNe laser was used as a coherent light source. The wavefront was expanded and filtered by a $15 \mu \mathrm{m}$ spatial filter. The fruit was illuminated directly by the filter without diffusers, as can be observed in the simplified scheme of Fig. 1. A 1 megapixel CMOS camera was used to record sequences of images.

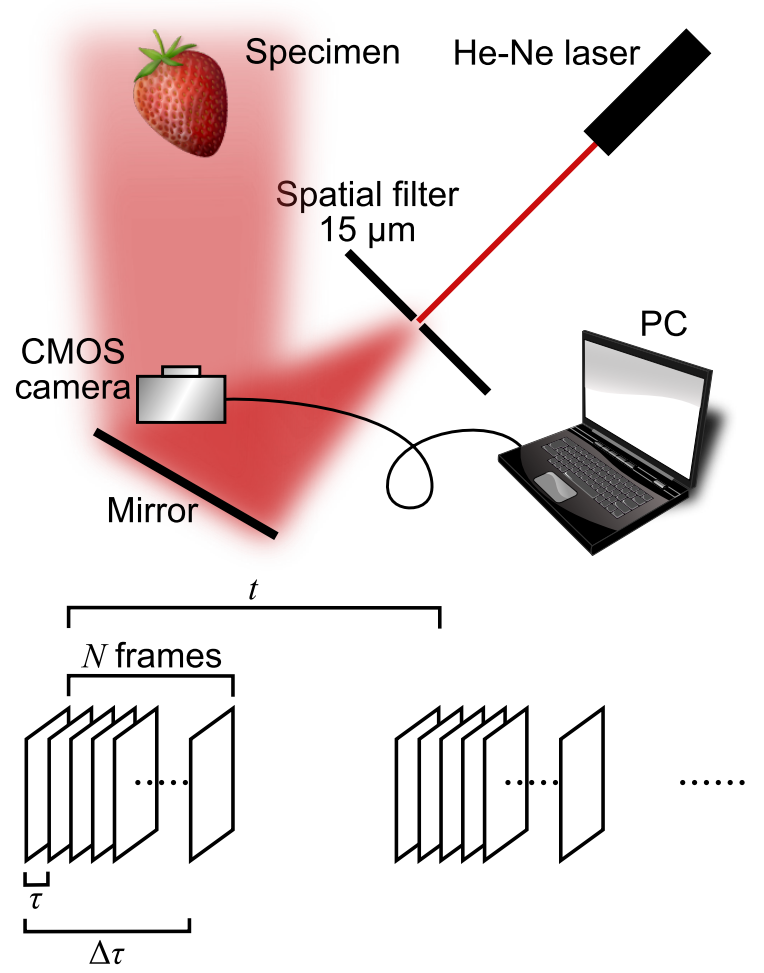

Figure 1. Simplified scheme of the optical set up and the temporal parameters involved during image acquisition

\subsection{Algorithm development}

For the analysis of the dynamic speckle patterns, we developed a simple processing algorithm using the MATLABß interface. It consists of a digital image capture routine and a data processing block of instructions, based on statistical analyses performed on the evolution of the dynamic speckle images. As a prerequisite, it is necessary to take $N$ photographs in a sequence having a well-defined time interval between them, $\tau$. From this statistical analysis, a mobility index (MI) is defined to represent the overall activity of the dynamic speckle pattern image sequence, as

$$
\mathrm{MI}=\frac{1}{m \times n} \sum_{k=1}^{N-1} \frac{1}{N-1} \sum_{i}^{m} \sum_{j}^{n}\left|I_{k+1}(i, j)-I_{k}(i, j)\right| .
$$

Stability of light intensity values is very important for a proper performance of the algorithm, since there is a direct correlation between the calculated MI and the reflected light intensity.

Before processing, photographs were converted into grayscale images and then transformed into numeric matrices, correlating each one of their elements to a pixel. The gray value of each pixel ranges from 0 to 255 , where 0 represents colour black and 255 white. With the information obtained from the difference of eq. (1) and using a simple subroutine it is possible to achieve a reconstruction of the fruit's image, which indicates the ripening state of each region of the fruit's surface. 


\subsection{Samples under study}

In this work we have studied four different strawberry samples (A, B, C and D from now on). Sample A was analyzed during $t=24 \mathrm{~h}$ acquiring two sequences of $N=10$ photographs with $\tau=1 \mathrm{~s}$ between them (see Fig. 1). For the rest of the samples, the observation period was fixed at $t=30 \mathrm{~min}$ taking also $N=10$ photographs and $\tau=1 \mathrm{~s}$. In this way, sample B was inspected during $87 \mathrm{~h}$, sample $\mathrm{C}$ during $82 \mathrm{~h}$ and sample $\mathrm{D}$ during $74 \mathrm{~h}$. Sample D was frozen at $-12{ }^{\circ} \mathrm{C}$ for $24 \mathrm{~h}$ previous to the experiment, in order to hasten the ripening process.

\section{RESULTS}

In the following sections we detail the results obtained from each sample.

\subsection{Sample A}

This sample was used as a test for the algorithm and allowed us to prove that the MI diminished appreciably from one day to the next. For this we calculated some statistical descriptors, such as average, mean, mode, etc., which are presented in Table 1. The MI value dropped from 1.44 to 0.89 .

Table 1. Statistical descriptors of sequences taken on first and second day for sample A.

\begin{tabular}{|l|l|l|}
\hline Descriptor & Day 1 & Day 2 \\
\hline Mean & 1.160 & 0.950 \\
\hline Standard error & 0.004 & 0.000 \\
\hline Median & 0 & 0 \\
\hline Mode & 0 & 0 \\
\hline Standard deviation & 1.962 & 1.685 \\
\hline Variance & 3.849 & 2.841 \\
\hline Range & 28 & 27 \\
\hline Min. & 0 & 0 \\
\hline Max. & 28 & 27 \\
\hline MI & $\mathbf{1 . 4 4 0}$ & $\mathbf{0 . 8 8 7}$ \\
\hline
\end{tabular}

Fig. 2 presents the image reconstruction of sample A which clearly shows that as cellular activity decayed, between the first and second day, the image definition deteriorated. This is in agreement with the diminishing of MI.
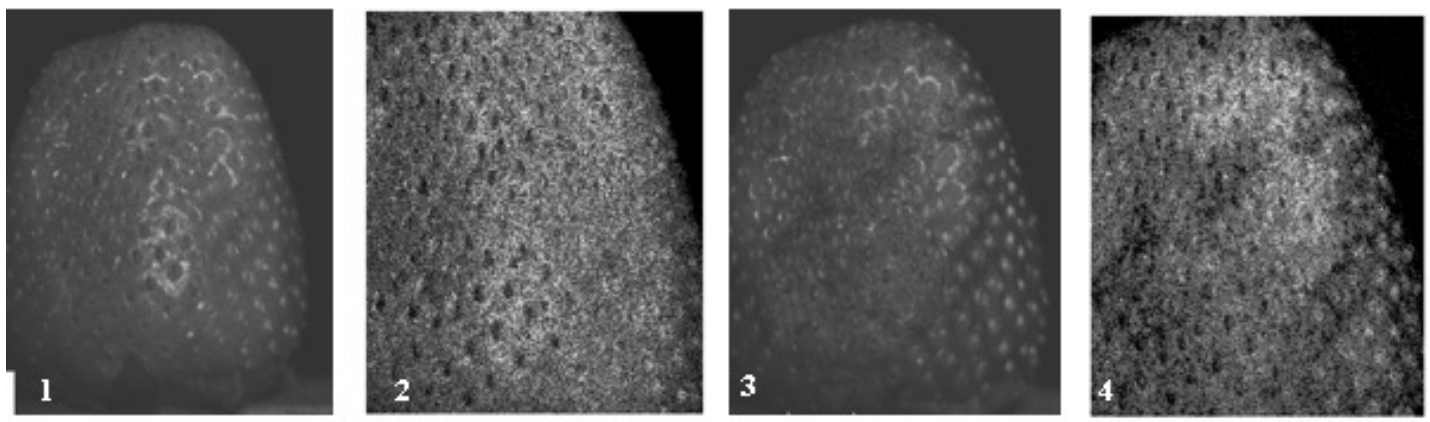

Figure 2. Sequence of photographs of a strawberry reconstructed by the algorithm, (1) image of the first day, (2) first day reconstruction, (3) image of the second day, (4) second day reconstruction. 


\subsection{Sample B}

The MI recorded for this sample ranged between 10.0 and 7.2. In Fig. 3 it is possible to observe a decreasing mobility as a function of time, which shows a slight fluctuation above and below the average trend curve.

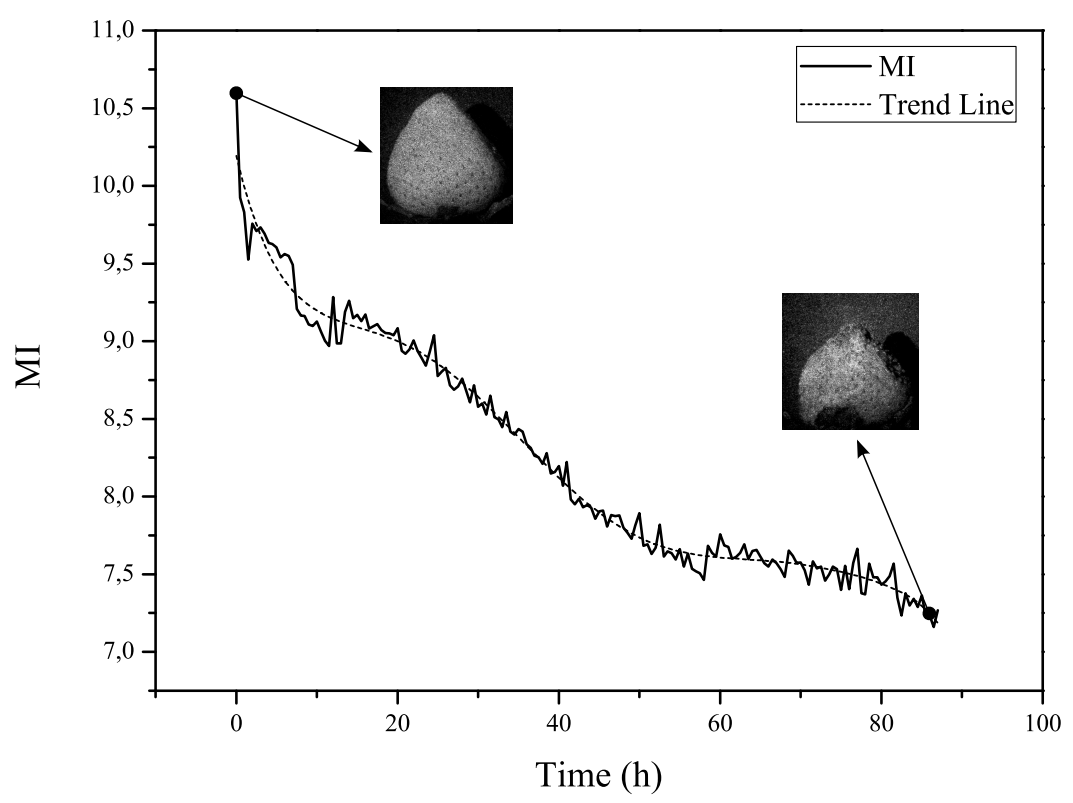

Figure 3. MI evolution of sample A over time and reconstructions of initial and final state images.

\subsection{Sample C}

For this strawberry, the MI recorded ranged between 5.0 and 1.6. The evolution of the mobility through the observation period is shown in Fig. 5. A black area can be seen in the bottom right region of the reconstructed

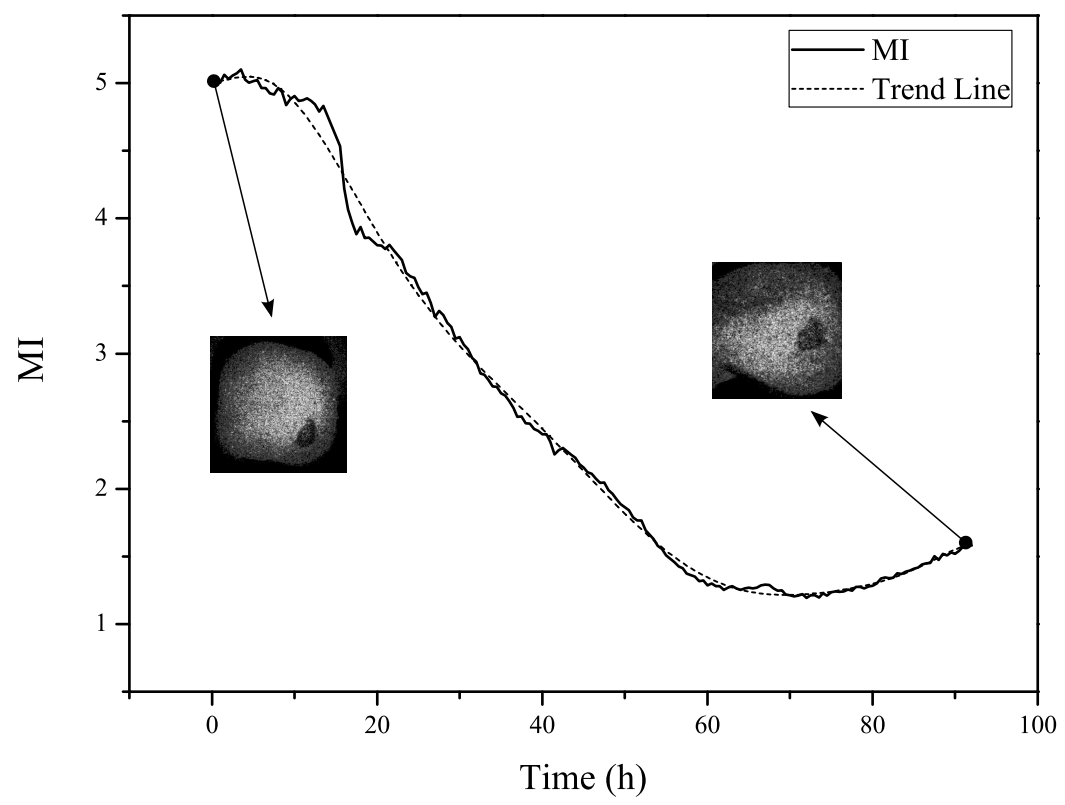

Figure 4. MI evolution of sample $\mathrm{C}$ over time and reconstructions of initial and final state images. 
image for the initial state of this strawberry. The black spot corresponds to a bruised area, where the registered local MI registered was nearly zero throughout the experiment, which is in accordance with the fact that the MI is lower than in sample B. We observed a constant MI diminution for approximately $60 \mathrm{~h}$, followed by a slight increment for longer times which is due to fungi activity as is detailed in Section 4.

\subsection{Sample D}

For this sample we obtained MI values ranging between 9.0 and 0.7. All data obtained is shown on Fig. 5 . MI presented an important decrease in the first $5 \mathrm{~h}$ period, followed by a decay without important random fluctuations above and below the average curve.

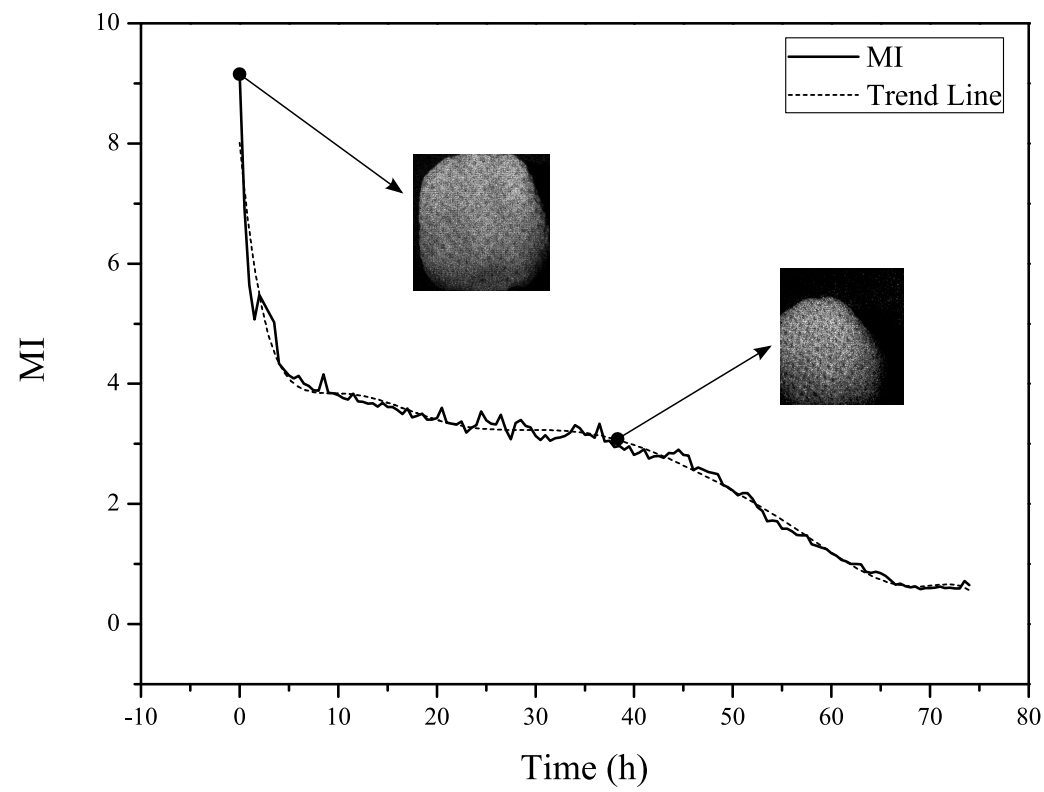

Figure 5. RMI evolution of sample $\mathrm{C}$ over time and reconstructions of initial and final state images.

\section{DISCUSSION}

Results obtained from all samples showed a typical response of ripening biological tissues, i. e. a MI diminution as the fruit matures. The distribution of values above and below the average curve is related to biospeckle pattern's random behavior. Sample C presented an increase in the mobility index for no apparent reason, approximately
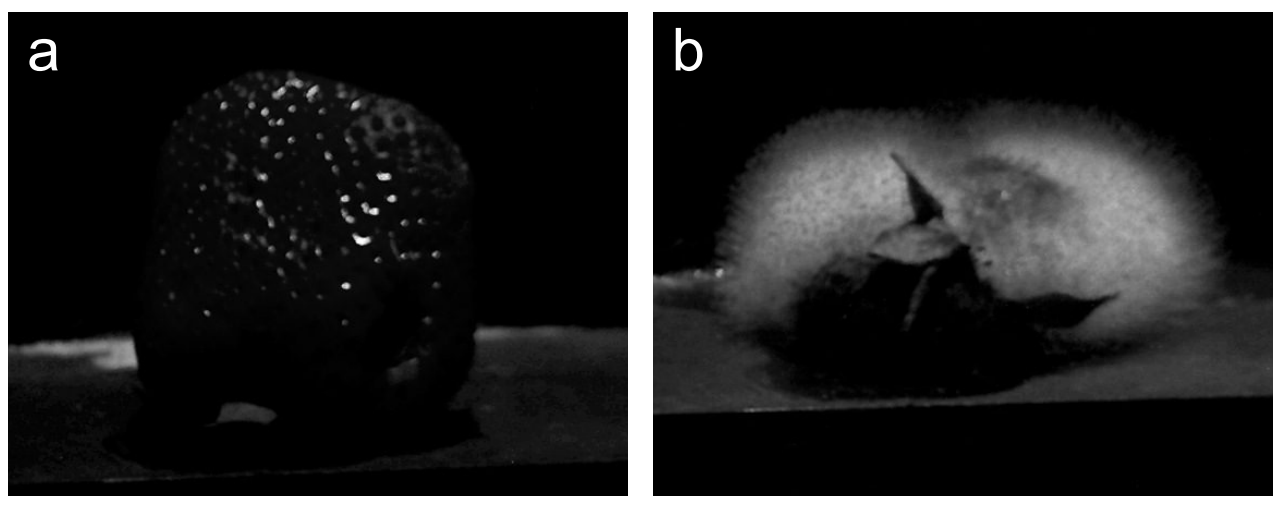

Figure 6. (1) Sample C at the beginning of the experiment (2) Sample B at the end of the experiment. 
$60 \mathrm{~h}$ after the start of the experiment. Under the same conditions, hours later, we noticed the formation of fungi colonies (see Fig. 6). These results allowed us to infer that the increase in the mobility of the dynamic speckle pattern was due to the activity of fungi growth. ${ }^{7}$

For sample D, cell mobility diminished faster than in the other samples. This was due to the damage inflicted to the cells when the fruit was frozen. Moreover, we attribute such high initial MI values to the fact that the sample was defrosting and that might have caused higher activity due to humidity condensation and subsequent evaporation on the fruit's surface.

\section{CONCLUSION}

This paper proposes a simple processing algorithm to analyse images obtained from biospeckle patterns, different from those documented in the available literature. This algorithms allows to achieve an image reconstruction, besides an index that represents cellular mobility in the sample under study. The application of this method shows that it's possible to define the fruit's conservation state from the study of its dynamic speckle patterns. All this procedure was verified by contrast with the methods specified in existing literature sources.

\section{ACKNOWLEDGMENTS}

This work was funded by joint projects from Universidad Tecnológica Nacional of Argentina and Universidade Da Coruña of Spain.

\section{REFERENCES}

[1] Rabal, H. J. and Braga, R. A., [Dynamic Laser Speckle and Applications], CRC Press Taylor \& Francis Group, Boca Raton Fl. 181-220 (2009).

[2] Lammertyn, J., Peirs, A., Baerdemaeker, J. D., and Nicolai, B., "Light penetration properties of nir radiation in fruits with respect to non-destructive quality assessment," Postharvest Biology and Technology 18, 121-132 (2000).

[3] Braga, R., Silva, B. O., Rabelo, G., Costa, R. M., Enes, A. M., Cap, N., Rabal, H., Arizaga, R., Trivi, M., and Horgan, G. W., "Reliability of biospeckle image analysis," Optics and Laser in Engineering 45, 390-395 (2007).

[4] Fujii, H., Nohira, K., Yamamoto, Y., Ikawa, H., and Ohura, T., "Evaluation of blood flow by laser speckle image sensing. part i," Applied Optics 26, 5321-5325 (1987).

[5] Arizaga, R., Cap, N., Rabal, H. J., and Trivi, M., "Display of local activity using dynamic speckle patterns," Opt. Eng. 41, 287-294 (2002).

[6] Aizu, Y. and Asakura, T., "Biospeckle phenomena and their application to the evaluation of blood flow," Opt. Laser Technol. 23, 205-219 (1991).

[7] Braga, R. A., Rabelo, G. F., Granato, L. R., Santos, E. F., Machado, J. C., Arizaga, R., Rabal, H. J., and Trivi, M., "Detection of fungi in beans by the laser biospeckle technique," Biosystems Engineering 91, 465-469 (2005). 